\title{
Fiber, the Ultimate Diet
}

\author{
Hildemar Dos Santos*1 and Natalie Gonzalez ${ }^{2}$ \\ ${ }^{1}$ Director of Preventive Care Program, Loma Linda University, USA \\ ${ }^{2}$ Loma Linda University, USA
}

*Corresponding author: Hildemar Dos Santos, Loma Linda University, USA, Email: hdossantos@llu.edu.

To Cite This Article: Hildemar Dos Santos. Fiber, the Ultimate Diet. Am J Biomed Sci \& Res. 2019 - 5(2). AJBSR.MS.ID.000896. DOI: 10.34297/ AJBSR.2019.05.000896.

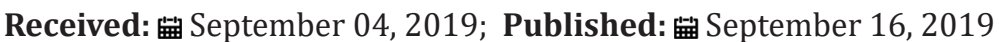

\section{Introduction}

Based on case studies in clinical settings, increasing the consumption of fiber will produce positive health outcomes. I remember one of my patients that had type 2 diabetes, hypertension, and a BMI over 30. He was taking insulin shots, metformin and a beta-blocker for blood pressure. After a period of two months on a high fiber diet ( 40 grams of fiber per day), he was able to drop insulin completely, drop to half his medication for diabetes and hypertension, and he lost 14 pounds. The advantage of this plan was that he was not on a diet. In fact, he was eating more than he used to before starting the fiber intervention.

Dietary fiber intake has many health benefits. With increased consumption, not only are there health-protective effects, but also disease-reversal benefits [1]. Dietary fiber can help with an array of diseases, such as: coronary heart disease, stroke, hypertension, diabetes type 2 , obesity, some cancers, and certain gastrointestinal diseases [1-3]. Dietary fiber influences these diseases through a variety of ways such as: improving serum lipoprotein values, lowering blood pressure, improving blood glucose control for diabetic individuals, aiding in weight loss and improving regularity [1].

Dennis Burkitt, also called the fiber doctor, used to illustrate the benefits of fiber by saying that the health of a nation could be estimated by considering the average size of the population's stools. Western countries that adopt a refined or very low fiber diet would have more intestinal diseases and colon cancer than countries where there was a consumption of high fiber foods. According to Burkitt, many gastrointestinal diseases such as diverticulosis and appendicitis were related to constipation because of a low fiber diet [4]. He also claimed that varicose veins, hemorrhoids and deep vein thrombosis were in the same way related to constipation and low fiber intake [5].

A recent meta-analysis displayed a significant dose-response relationship between fiber and coronary heart disease. With increasing amounts of fiber, there was a greater protection against this disease [6]. In addition, another study showed similar results, along with an association with lower inflammation levels with increased amounts of fiber in one's diet. High inflammation levels can also contribute to cardiovascular risk [7]. Dietary fiber influences cardiovascular health, as well as glucose control because of the viscosity of the fiber. When the fiber becomes viscous, it thickens the contents in the lumen of the intestine, which slows the absorption of nutrients, such as cholesterol and sugar. The viscosity also disrupts bile salt reabsorption, which leads to lower cholesterol levels. Furthermore, it can lower glucose response, which can assist in reduced insulin stimulation [8].

Dietary fiber also shows protective benefits towards colon cancer. A meta-analysis showed that there was a reduction in colon cancer by $10 \%$ when 10 grams of fiber were incorporated daily. This is mainly attributed to increased fecal bulk and decreased transit time. Through this mechanism, colorectal epithelium is not only exposed to lower concentrations of carcinogens, but for even a shorter period. In addition, short-chain fatty acids, which are produced by fiber in the gut, are beneficial to colonocytes, thus enhancing its health and assisting in colorectal cancer prevention [2]. Dietary fiber also has an influence on weight [1-3]. Certain fibers, such as beta-glucan, enhance postprandial satiety through physical and chemical properties [3].

Finally, a most recent meta- analysis, conducted under the request of the World Health Organization, showed results that confirmed the benefits of fiber in the prevention of chronic diseases [9]. The study evaluated 185 prospective studies and 58 clinical trials, and it suggested a $15-30 \%$ decrease in all-cause mortality, cardiovascular mortality, incidence of coronary heart disease, incidence and mortality of stroke, type 2 diabetes and colorectal cancer. The amount of fiber that produced those benefits were between 25-29 grams. The authors suggested that there is a doseresponse relationship for the benefits, and higher intakes could produce higher benefits mostly on cardiovascular diseases, type 2 diabetes, colorectal cancer and even breast cancer [9]. 
Despite all these benefits from dietary fiber, children and adults in the US are consuming, on average, less than half of the recommended amount [1]. The recommended amount of fiber, based off the Adequate Intake, is 14 grams of total fiber per $1,000 \mathrm{kcal}$, or 25 grams for adult women and 38 grams for adult men [1,2]. Fiber can come from a variety of plant sources, such as: fruits, vegetables, whole grains, legumes, nuts, and seeds [2]. Overall, dietary fiber shows favorable results to health and can influence disease reversal and prevention [1-9] Therefore, both the prevention and treatment of the above-mentioned diseases should include the increase of fiber intake, as evidenced by science. Also, because it is generally of lower cost and high applicability to populations.

\section{References}

1. Anderson JW, Baird P, Davis RH Jr, Ferreri S, Knudtson M, et al. (2009) Health benefits of dietary fiber. Nutrition reviews 67(4): 188-205

2. Dahl WJ, Stewart ML (2015) Position of the Academy of Nutrition and Dietetics: Health implications of dietary fiber. Journal of the Academy of Nutrition and Dietetics 115(11): 1861-1870.
3. Fuller S, Beck E, Salman H, Tapsell L (2016) New horizons for the study of dietary fiber and health: A review. Plant Foods for Human Nutrition (Dordrecht, Netherlands) 71(1): 1-12.

4. Burkitt DP (1980) Eat Right-To Stay Healthy and Enjoy Life More: How Simple Diet Changes Can Prevent Many Common Diseases.

5. Burkitt DP (1972) Varicose veins, deep vein thrombosis, and haemorrhoids: epidemiology and suggested aetiology. British Medical Journal 2(5813): 556-561.

6. Wu YH, Qian YF, Pan YW, Li P, Yang J, et al. (2015) Association between dietary fiber intake and risk of coronary heart disease: A meta-analysis. Clin Nutr 34(4): 603-611.

7. Ning H, Van Horn L, Shay CM, Lloyd Jones DM (2014) Associations of dietary fiber intake with long-term predicted cardiovascular disease risk and C-reactive protein levels (from the National Health and Nutrition Examination Survey Data [2005-2010]). Am J Cardiol 113(2): 287-291.

8. Dai FJ, Chau CF (2017) Classification and regulatory perspectives of dietary fiber. J Food Drug Anal 25(1): 37-42.

9. Reynolds A, Mann J, Cummings J, Winter N, Mete E, et al. (2019) Carbohydrate quality and human health: A series of systematic reviews and meta-analyses. Lancet 393(10170): 434-445. 